\title{
How the COVID-19 pandemic changed treatment of severe aortic stenosis: a single cardiac center experience
}

\author{
Bartlomiej Perek ${ }^{1}$, Anna Olasinska-Wisniewska ${ }^{1}$, Marcin Misterski ${ }^{1}$, Mateusz Puslecki ${ }^{1,2}$, Marek Grygier ${ }^{3}$, \\ Piotr Buczkowski ${ }^{1}$, Maciej Lesiak ${ }^{3}$, Tomasz Stankowski ${ }^{4}$, Lukasz Szarpak ${ }^{5,6}$, Kurt Ruetzler ${ }^{7}$, Oguz Turan $^{7}$, \\ Marek Jemielity ${ }^{1}$
}

${ }^{1}$ Department of Cardiac Surgery and Transplantology, Poznan University of Medical Sciences, Poznan, Poland; ${ }^{2}$ Department of Medical Rescue, Poznan University of Medical Sciences, Poznan, Poland; ${ }^{3} 1^{\text {st }}$ Department of Cardiology, Poznan University of Medical Sciences, Poznan, Poland; ${ }^{4}$ Department of Cardiac Surgery, Sana-Herzzentrum Cottbus, Cottbus, Germany; ${ }^{5}$ Bialystok Oncology Center, Bialystok, Poland; ${ }^{6}$ Maria SklodowskaCurie Medical Academy in Warsaw, Warsaw, Poland; ${ }^{7}$ Department of Outcomes Research, Anesthesiology Institute, Cleveland Clinic, OH, USA

Contributions: (I) Conception and design: B Perek, A Olasinska-Wisniewska, L Szarpak, M Jemielity; (II) Administrative support: M Grygier, M Lesiak, M Jemielity; (III) Provision of study materials or patients: B Perek, A Olasinska-Wisniewska, M Misterski, M Puslecki; (IV) Collection and assembly of data: B Perek, M Misterski, M Puslecki, M Grygier, P Buczkowski, M Lesiak, T Stankowski, L Szarpak; (V) Data analysis and interpretation: B Perek, A Olasinska-Wisniewska, P Buczkowski, T Stankowski, L Szarpak, K Ruetzler, O Turan; (VI) Manuscript writing: All authors; (VII) Final approval of manuscript: All authors.

Correspondence to: Bartlomiej Perek, MD, PhD. Department of Cardiac Surgery and Transplantology, Poznan University of Medical Sciences, 1/2 Dluga Street, 61-848 Poznan, Poland. Email: bperek@yahoo.com.

Background: Currently, two effective therapeutic options for severe aortic stenosis (AS) are available, one catheter-based [transcatheter aortic valve implantation (TAVI)], the other open surgical approach [surgical aortic valve replacement (SAVR)]. The COVID-19 pandemic has limited the availability of medical procedures. The purpose of this cross-sectional study was to assess if this pandemic had any impact on the treatment strategy of severe AS in a single cardiac center.

Methods: This study involved AS patients treated in 3-month periods (February through April) over 3 consecutive years 2018, 2019 [defined as $\operatorname{COV}(-)$ group] and $2020[\mathrm{COV}(+)]$. We assessed if there were any differences regarding patients' clinical profile, applied therapeutic method, procedure complexity and early clinical outcomes.

Results: In the years 2018 through 2019, approximately 50\% of AS patients were treated classically (SAVR) while in 2020 this rate dropped to $34 \%$. The preoperative clinical characteristic of TAVI subjects was comparable irrespective of the year. Regarding SAVR, more patients in $\mathrm{COV}(+)$ underwent urgent and more complex procedures. More of them were found in NYHA class III or IV, and had lower left ventricular ejection fraction (LVEF) $(51.9 \% \pm 14.4 \%$ vs. $58.3 \% \pm 8.1 \% ; \mathrm{P}=0.021)$ than in $\mathrm{COV}(-)$ individuals. During the pandemic, a change in applied therapeutic methods and differences in patients' clinical profile did not have an unfavorable impact on in-hospital mortality (2.0\% before $v s .3 .6 \%$ during pandemic) and morbidity. Of note, intubation time and in-hospital stay were significantly shorter $(\mathrm{P}<0.05)$ in 2020 (4.2 hours and 7.5 days $)$ than in the previous years (7.5 hours and 9.0 days, respectively).

Conclusions: The coronavirus pandemic has changed substantially the management of severe AS. The shift into less invasive treatment method of AS patients resulted in shortening of in-hospital stay without compromise of short-term outcomes.

Keywords: Aortic stenosis (AS); transcatheter aortic valve implantation (TAVI); aortic valve replacement; COVID-19 pandemic

Submitted Oct 07, 2020. Accepted for publication Dec 13, 2020.

doi: $10.21037 /$ jtd-20-3025

View this article at: http://dx.doi.org/ 10.21037/jtd-20-3025 


\section{Introduction}

Severe aortic valve stenosis (AS) is one of the most prevalent forms of acquired valvular heart disease $(1,2)$. Currently two therapeutic options for AS are available; transcatheter aortic valve implantation (TAVI), a percutaneous minimally invasive method, and surgical aortic valve replacement (SAVR) which is performed with cardio-pulmonary bypass (CPB) (3). Although SAVR can be done from a partial sternotomy which is considered as less invasive access, the patients still require both meticulous care and treatment soon after surgery in the intensive care units (ICUs) (4). In contrast, TAVI patients are usually transferred to cardiac ICUs which are a part of the cardiologic wards (5). The coronavirus (SARS$\mathrm{CoV}$-19) pandemic has created an enormous challenge for all health care systems around the world. This is especially true for ICUs as many infected patients develop hypoxia and require intense respiratory support $(6,7)$. Certain multispecialty hospitals were converted into COVID-19 treatment centers with the intention being to exclusively treat coronavirus patients. Consequently, the majority of ICU beds in these hospitals were occupied by the victims of the pandemic. In addition, extraordinary financial resources were allocated to the diagnosis and management of COVID-19 patients (8). As many countries also had drastic lockdowns, a global economic regression followed (9). Due to the state of emergency, the rate of elective procedures decreased substantially including cardiac surgical operations (10). Furthermore, patients with mild symptoms of cardiovascular diseases preferred to stay at home instead. For stable individuals with valvular heart disease postponing surgery and optimal pharmacotherapy were recommended by some national cardiac societies (11). A number of cardiac surgery departments limited their operations to cases such as aortic dissection in young patients or urgent coronary artery bypass grafting (CABG) that could not be fixed through percutaneous coronary interventions (PCIs) (10,12). Furthermore, less invasive therapeutic options, even if not the method of choice, were used to reduce the length of both intensive treatment and total inhospital stay $(10,12)$.

Therefore, the purpose of our cross-sectional study was to determine if the COVID-19 pandemic had any impact on management, the clinical profile and early outcomes of patients treated for severe AS. We present the following article in accordance with the STROBE reporting checklist (available at http://dx.doi.org/10.21037/jtd-20-3025).

\section{Methods}

\section{Patients}

The periods of interest confined to 90 days, from February 1st to April 30th, during 3 consecutive years, 2018-2020. All patients were treated invasively for severe AS in the Department of Cardiac Surgery and Transplantology in Poznan (Poland). Individuals who required simultaneous CABG were also included. The following exclusion criteria such as concomitant severe other valves defects, aortic diseases, significant aortic valve insufficiency and infective endocarditis were applied.

\section{Ethical statement}

The study was conducted in accordance with the Declaration of Helsinki (as revised in 2013). As this study is a retrospective analysis of routine patients treated in the Department of Cardiac Surgery and Transplantology, approval of the Bioethical committee was not necessary and the Institutional Review Board waived the requirement of individual patient consent.

\section{Preoperative period}

Patients diagnosed with severe AS were given either TAVI or SAVR after the recommendations of the local heart team that consisted of specialists in echocardiography, interventional cardiology and cardiac surgery. The patients' medical history such as clinical presentation, findings in the imaging studies such as transthoracic echocardiography (TTE), contrast enhanced computed tomography with off-line reconstructions and coronary angiography were analyzed. Patients not suitable or disqualified from SAVR were conserved as candidates for TAVI. Thus, the latter method was reserved mostly for elderly subjects with serious comorbidities being at moderate or high risk for early mortality and morbidity. In cases with critical stenosis in the coronary arteries, PCI was carried out a few weeks before TAVI while in SAVR, CABG was done simultaneously.

\section{TAVI}

TAVI procedures were described earlier and were usually done in deep sedation, preferred, or general anesthesia with a percutaneous femoral approach (13). Routinely, a temporary pacemaker to the right ventricle was deployed through the femoral vein. Once the prosthesis was correctly 
positioned and expanded, the contrast medium was injected to check for the presence of a paravalvular leak. In addition, echocardiography was done to check the function of the implanted bioprosthesis.

\section{$S A V R$}

SAVR individuals were treated either electively or urgently. Operations were defined as urgent if the patients had to wait for aortic valve surgery in the hospital whereas elective cases stayed at home for usually a few weeks or even months after the final diagnosis had been established.

All surgeries were performed through partial or full median sternotomy with the use of CPB in moderate hypothermia $\left(28\right.$ to $30{ }^{\circ} \mathrm{C}$ ) and cardioplegic arrest. If the patients had to undergo simultaneous CABG, distal anastomoses of free grafts, usually segments of saphenous vein, were done first after aortic cross-clamping and cardioplegic arrest. The valve replacement and left internal thoracic artery anastomosis followed. After resuming the coronary flow, proximal anastomoses of the free grafts were performed on the partially clamped ascending aorta. In general, younger patients received mechanical prostheses while the elderly received bioprostheses. Of note, the final choice was always left to the patients' decision.

\section{Postoperative period}

The selected individuals after TAVI (with intraprocedural complications or these considered preoperatively as very high risk) and all patients who got SAVR were transferred to the postoperative ICU, intubated and mechanically ventilated. According to the hospital rules, patients in stable clinical status after ICU stay were further treated at either the cardiac surgery (SAVR) or cardiology (TAVI) ward. The subjects after open surgical operations were usually discharged to rehabilitation centers whereas those following percutaneous interventions usually were sent home directly.

\section{Parameters included}

The following variables were analyzed:

* Demographic: age, rate of elderly (>75 years), gender;

* Clinical: priority of surgery (elective/urgent), functional status according to New York Heart Association (NYHA) classification, concomitant disorders such as arterial hypertension (AH), hyperlipidemia, diabetes mellitus, peripheral vascular disease, chronic obstructive pulmonary disease, chronic kidney disease;

* Results of the imaging studies (echocardiography or coronary angiography);

* A risk of intervention on the aortic valve stratified by means of EuroSCORE II calculator.

Postoperative in-hospital course, including mortality and morbidity was also analyzed. Moreover, a length of endotracheal intubation (if applicable), ICU and in-hospital stays were evaluated. Additionally, the following serious adverse events were taken into account:

* Myocardial infarction (defined if all conditions such as significant increase in troponin I concentration, ischemic changes in electrocardiogram and new local disturbances in myocardial contractility were fulfilled simultaneously);

* Atrioventricular block (AVB) requiring permanent pacemaker implantation;

* Renal failure treated by means of renal replacement therapy [continuous veno-venous hemodialysis (CVVHD)];

* Respiratory failure if patients had to be intubated longer than 12 hours or be reintubated;

* Stroke [confirmed in neurological (specialist consultation) and imaging examinations (computed tomography or magnetic resonance imaging)];

* Bleeding from surgical access (TAVI) or bleeding/ tamponade requiring chest re-exploration;

* Deep surgical site infection (DSSI) treated with repeat sternal reosteosynthesis (after SAVR);

* Peripheral TAVI access serious complications (e.g., artery dissection, false aneurysm) if vascular open or intravascular interventions were necessary.

\section{Data analysis}

First, all continuous variables were checked for normality by means of the Shapiro-Wilk test. If the variables satisfied the normal distribution criteria, they are presented as means with standard deviation (SD) and then compared with the use of ANOVA followed by the Scheffe Post Hoc test. Otherwise, they are expressed as the medians with interquartile ranges $\left(25^{\text {th }}-75^{\text {th }}\right.$ percentile). Categorical data are presented as the numbers (n) with percentages (\%). The aforementioned variables were analyzed with the use of the Kruskal-Wallis test followed by multiple comparisons of the ranks. In addition, we compared the most basic variables 


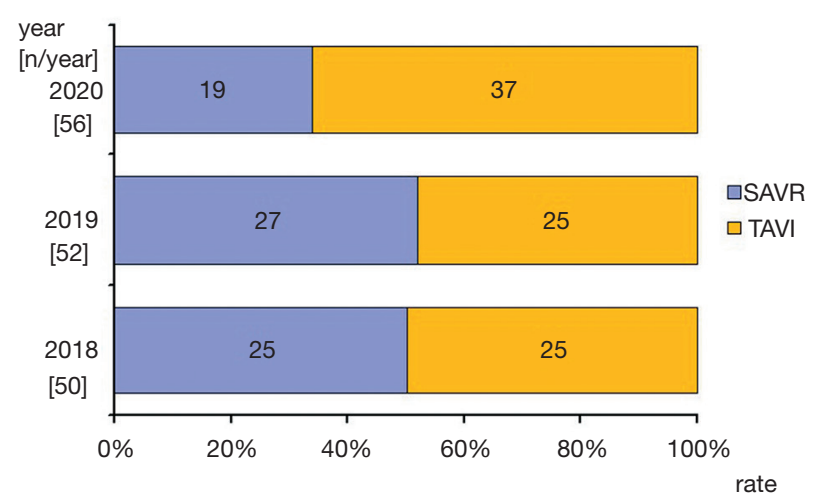

Figure 1 Applied method of treatment of severe AS. AS, aortic stenosis; SAVR, surgical aortic valve replacement; TAVI, transcatheter aortic valve implantation.

describing patients who underwent surgery for significant AS before [years 2018 to 2019; COV(-) group] and during COVID-19 pandemic [year 2020; COV(+) group]. To compare $\operatorname{COV}(-)$ with $\mathrm{COV}(+)$ subjects, unpaired t-test, Mann-Witney $U$ and $\chi^{2}$ tests with or without Yates correction were employed. A P value below 0.05 was considered as statistically significant. All calculations were done in the Statistica 10.0 software package (StatSoft, Tulsa, OK, USA).

\section{Results}

\section{Applied therapeutic method}

A total number of AS patients treated in our center were similar over the 3 consecutive years and ranged from 50 in 2018 to 56 in the corresponding months of 2020. However, a shift from SAVR to minimally invasive TAVI procedures was seen. In the years 2018 through 2019, approximately $50 \%$ of patients were treated classically (AVR; 50\% in 2018 and $52 \%$ in 2019) while after the COVID-19 pandemic outbreak this rate dropped to $34 \%$. The detailed numbers of AS patients treated in the years 2018 through 2020 are presented graphically (Figure 1).

\section{Preoperative patients' characteristics}

The preoperative clinical characteristics were similar in TAVI group irrespective of the year. The only exception was a higher prevalence of patients after neurological events [either transient ischemic attack (TIA) or cerebrovascular accidents (CVAs)] in 2018 (7/25, 28.0\%) compared to 2020
(2/37, 5.4\%; $\mathrm{P}=0.035)$.

Post hoc analysis (of normally distributed continuous data) or multiple comparisons between ranks (for the other variables) did not reveal any differences in SAVR patients treated before pandemic outbreak, specifically between 2018 and 2019. Therefore, they were entered into the further analysis as an one group [COV(-)].

In contrast, substantial differences in the clinical profile of $\operatorname{COV}(-)$ and $\operatorname{COV}(+)$ individuals were noted. Firstly, more than $50 \%$ of patients who underwent SAVR during the pandemic were found in NYHA classes III and IV as well as more frequently presenting with symptoms of coronary artery disease (CAD). Although post hoc analysis of the mean age of SAVR individuals did not reveal any differences, the rate of elderly patients (75 years and more) decreased significantly in 2020 [COV(+) group]. The other findings of the preoperative clinical evaluations are outlined in Table 1.

In the preoperative TTE, left ventricular (LV) performance was worse in $\mathrm{COV}(+)$ compared to those operated earlier, the $\mathrm{COV}(-)$ group. In the $\mathrm{COV}(-)$ group mean left ventricular ejection fraction (LVEF) was markedly lower $(\mathrm{P}=0.021)$ and $\mathrm{LV}$ end-diastolic dimension (LVEDd) larger $(\mathrm{P}=0.016)$ than in $\mathrm{COV}(-)$. Additionally, significantly more subjects in $\mathrm{COV}(+)(\mathrm{P}=0.023)$ presented markedly impaired LV systolic function (LVEF below 30\%). More echocardiographic data are listed in Table 2.

No comparison between TAVI and SAVR patients regarding preoperative demographics and clinical status was performed because it was beyond the scope of this study.

\section{Priority of surgery and SAVR details}

Regarding SAVR patients, more of them required urgent treatment as well as simultaneous CABG in 2020 than previously. However, the number of implanted aortocoronary grafts were similar in the consecutive years. Selected data regarding open surgery are outlined in Table 3. In general, $\operatorname{COV}(-)$ subjects were of lower risk than $\mathrm{COV}(+)$ individuals.

\section{Postoperative course}

Overall, short-term mortality defined as all deaths that occurred within the first 30 days following procedure irrespective of patient stay (hospital, rehabilitation center, home) was $2.5 \%(\mathrm{n}=4)$, two fatal cases in both subgroups. Mortality rate among $\mathrm{COV}(-)$ individuals was $2.0 \%$ 
Table 1 Demographic and preoperative clinical data

\begin{tabular}{|c|c|c|c|c|c|c|}
\hline Groups* & \multicolumn{2}{|c|}{ All patients } & \multicolumn{2}{|c|}{ TAVI } & \multicolumn{2}{|c|}{ SAVR } \\
\hline Age (years) & $71.8 \pm 9.7$ & $71.4 \pm 10.2$ & $78.2 \pm 7.5$ & $75.6 \pm 6.1$ & $66.3 \pm 9.2$ & $63.5 \pm 13.2$ \\
\hline$>75$ years & $52(51.0)$ & $28(50.0)$ & $38(76.0)$ & 27 (73.0) & $14(26.9)$ & $1(5.3)^{\#}$ \\
\hline Gender (F/M) & $59(57.8) / 43$ (42.2) & $32(57.1) / 24(42.9)$ & $29(58.0) / 21(42.0)$ & $20(54.1) / 17(45.9)$ & $30(57.7) / 22(42.3)$ & $12(63.2) / 7(36.8)$ \\
\hline Obesity $^{1}$ & $21(20.6)$ & $13(23.2)$ & $14(28.0)$ & $10(27.0)$ & $7(13.5)$ & $3(15.8)$ \\
\hline $\mathrm{AH}$ & 73 (71.6) & $44(78.6)$ & $43(86.0)$ & $31(83.8)$ & $30(57.7)$ & $13(68.4)$ \\
\hline $\mathrm{HL}$ & $26(25.5)$ & $23(41.1)^{\#}$ & $13(26.0)$ & $13(25.0)$ & $13(25.0)$ & $10(52.6)^{\#}$ \\
\hline COPD & $6(5.9)$ & 7 (12.5) & $3(6.0)$ & $6(16.2)^{\#}$ & $3(5.8)$ & $1(5.3)$ \\
\hline TIA/CVA & $11(10.8)$ & $2(3.6)$ & $9(18.0)$ & $2(5.4)$ & $2(3.8)$ & $0(0.0)$ \\
\hline CAD & $44(43.1)$ & $29(51.8)$ & $25(50.0)$ & $16(43.2)$ & $19(36.5)$ & $13(68.4)^{\#}$ \\
\hline Prev. MI & $19(18.6)$ & $13(23.2)$ & $14(28.0)$ & $9(24.3)$ & $5(9.6)$ & $4(21.1)$ \\
\hline $\mathrm{PCl}$ in hist. & $25(24.5)$ & 19 (33.9) & $19(38.0)$ & $14(37.8)$ & $6(11.5)$ & $5(26.3)$ \\
\hline $\mathrm{RF}^{3}$ & $31(30.4)$ & $27(48.2)^{\#}$ & $28(56.0)$ & $24(64.9)$ & $3(5.8)$ & $3(15.8)$ \\
\hline$E S \|^{4}(\%)$ & 1.70 (1.21-2.99) & $2.68^{\#}(1.72-3.56)$ & $2.80(1.78-4.66)$ & $3.09(2.04-5.30)$ & $1.21(0.93-1.59)$ & $1.84^{\#}(1.11-3.35)$ \\
\hline
\end{tabular}

*, continuous data are expressed as the means \pm SD or the medians with interquartile ranges $\left(25^{\text {th }}-75^{\text {th }}\right.$ percentile) while categorical as the numbers (n) with percentages (\%); ", $\mathrm{P}<0.05 \operatorname{COV}(-)$ vs. $\operatorname{COV}(+)$ groups; bolded data are of statistical significance. ${ }^{1}$, defined if BMI exceeded $30 \mathrm{~kg} / \mathrm{m}^{2} ;{ }^{2}$, if glomerular filtration rate (eGFR) was below $60 \mathrm{~mL} / \mathrm{kg} / 1.73 \mathrm{~m}^{2} ;{ }^{3}$, it refers only to the arteries of the lower extremities; ${ }^{4}$, logistic EuroSCORE II stratifies risk of early mortality (up to 30 days following intervention). TAVI, transcatheter aortic valve implantation; SAVR, surgical aortic valve replacement; COV, patients treated before (-) or during COVID-19 (+) pandemic; F, female; M, male; NYHA, New York Heart Association class; AH, arterial hypertension; HL, hiperlipidemia; COPD, chronic obstructive pulmonary disease; DM, diabetes mellitus; PVD, peripheral vascular disease; TIA, transient ischemic attack; CVA, cerebrovascular accident (brain stroke); CAD, coronary artery disease; MI, myocardial infarction; $\mathrm{PCl}$, percutaneous coronary intervention; RF, renal failure; ES, EuroSCORE; SD, standard deviation; BMI, body mass index.

while $3.6 \%$ in $\operatorname{COV}(+)$ group (ns). Taking into account a method of aortic valve implantation, single SAVR patients died either in the years 2018-2019 or in 2020 (both had combined procedures, SAVR completed by CABG) whereas all TAVI subjects $(\mathrm{n}=2)$ before SARS-CoV-2 pandemic. The reasons of death myocardial ischemia-induced low cardiac output syndrome (LCOS) $(\mathrm{n}=2$; on 3 rd and 5 th postoperative days) in SAVR group whereas fatal bleeding due to perforation of left ventricle (intraoperative death) and LCOS preceding multi-organ failure (MOF) in TAVI subjects.

The other postprocedural serious adverse events are summarized in Table 4. Of note, the rate of them was comparable in the consecutive years in TAVI group $(28 \%$ in $2018,36 \%$ in 2019 and $24 \%$ in 2020; ns) while in SAVR subset it was significant higher $(\mathrm{P}=0.048)$ in $2020(42 \%)$ than in the years 2018-2019 (19\%).

Additionally, comparison of intubation time and inhospital stay revealed their median times for of all patients irrespective of valve implantation technique was significantly shorter in the year of SARS-CoV-2 pandemic than previously (see Figure 2). It probably resulted from higher contribution of TAVI procedures after pandemic outbreak. Detailed analysis of the aforementioned variables of the postoperative course were comparable within both subgroups in the consecutive years (SAVR $2018=$ SAVR $2019=$ SAVR 2020; TAVI $2018=$ TAVI $2019=$ TAVI 2020) but notable they were always markedly shorter in TAVI 
Table 2 Preoperative echocardiographic findings

\begin{tabular}{|c|c|c|c|c|c|c|}
\hline Groups* & \multicolumn{2}{|c|}{ All patients } & \multicolumn{2}{|c|}{ TAVI } & \multicolumn{2}{|c|}{ SAVR } \\
\hline $\mathrm{LA}(\mathrm{mm})$ & $40.7 \pm 6.8$ & $44.1 \pm 7.2^{\#}$ & $44.5 \pm 6.8$ & $45.7 \pm 6.5$ & $37.1 \pm 4.7$ & $41.6 \pm 7.7^{\#}$ \\
\hline LVEDd (mm) & $45.9 \pm 8.2$ & $49.0 \pm 8.2^{\#}$ & $44.3 \pm 9.4$ & $45.1 \pm 8.9$ & $47.5 \pm 6.4$ & $51.3 \pm 7.0^{\#}$ \\
\hline IVSd (mm) & $15.0 \pm 3.1$ & $13.9 \pm 2.6^{\#}$ & $13.5 \pm 2.3$ & $13.3 \pm 1.4$ & $16.3 \pm 2.2$ & $15.2 \pm 1.7^{\#}$ \\
\hline $\mathrm{RV}(\mathrm{mm})$ & $30.0 \pm 4.3$ & $32.1 \pm 5.1^{\#}$ & $29.8 \pm 4.7$ & $29.4 \pm 5.3$ & $30.2 \pm 3.8$ & $33.7 \pm 4.4^{\#}$ \\
\hline LVEF (\%) & $56.8 \pm 8.0$ & $51.9 \pm 12.8^{\#}$ & $55.1 \pm 8.5$ & $52.3 \pm 11.9$ & $58.3 \pm 8.1$ & $51.9 \pm 14.4^{\#}$ \\
\hline LVEF below $30 \%$ & $3(2.9)$ & $6(5.9)$ & $2(4.0)$ & $2(5.4)$ & $1(1.9)$ & $4(21.1)^{\#}$ \\
\hline PPG (mmHg) & $90.6 \pm 24.9$ & $84.3 \pm 19.6$ & $89.4 \pm 22.6$ & $85.7 \pm 22.2$ & $91.8 \pm 27.0$ & $81.5 \pm 15.2^{\#}$ \\
\hline
\end{tabular}

*, continuous data (as normally distributed) are presented as the means \pm SD whereas categorical data as the numbers ( $\mathrm{n}$ ) with percentages (\%); ", $\mathrm{P}<0.05 \operatorname{COV}(-)$ vs. $\operatorname{COV}(+)$; bolded data are of statistical significance. TAVI, transcatheter aortic valve implantation; SAVR, surgical aortic valve replacement; COV $(-) /(+)$, patients treated before $(-)$ or during (+) SARS-CoV-2 pandemic; LA, left atrial; LVEDd, left ventricular end-diastolic dimension; IVSd, interventricular septum thickness in diastole; LVPWd, left ventricular posterior wall thickness in diastole; RV, right ventricular; LVEF, left ventricular ejection fraction; PPG, peak pressure gradient; MPG, mean pressure gradient; SD, standard deviation.

Table 3 Details of SAVR

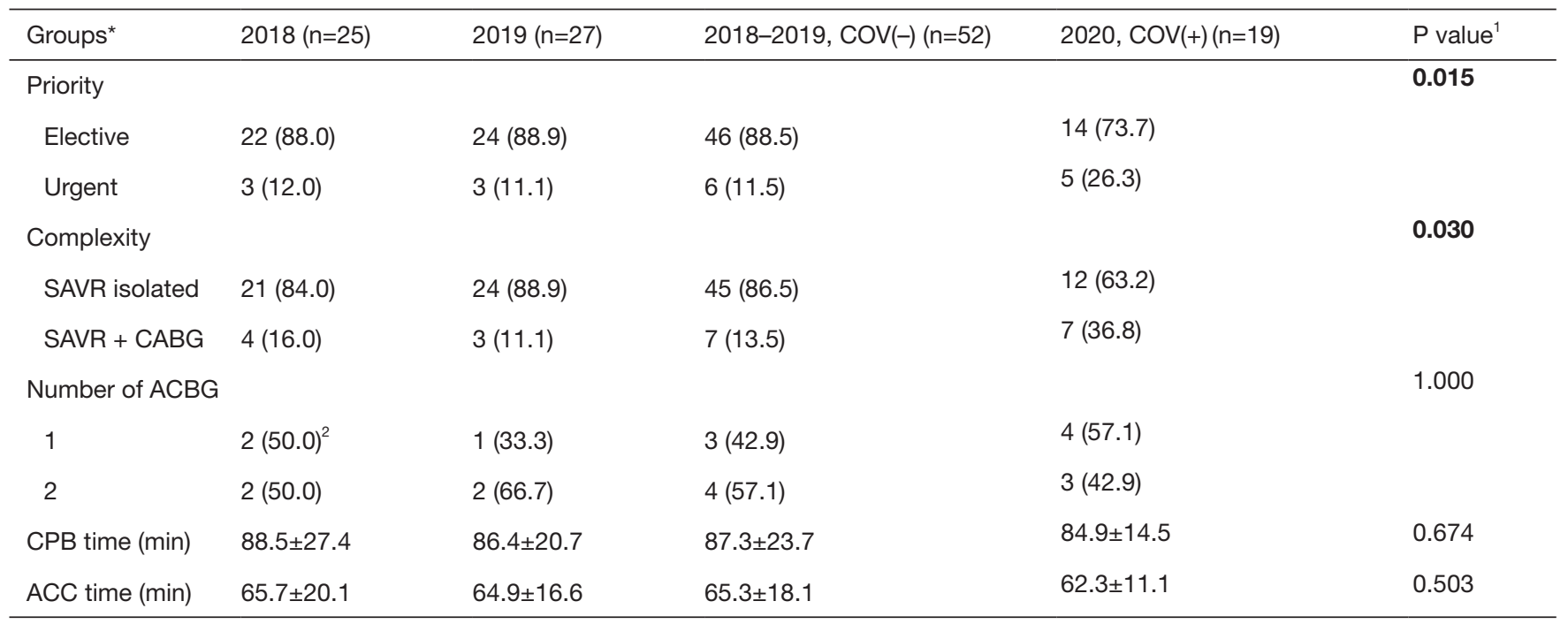

*, continuous data are expressed as the means $\pm \mathrm{SD}$ while categorical ones as the numbers $(\mathrm{n})$ with percentages $(\%) .{ }^{1}, \mathrm{P}<0.05$ $\operatorname{COV}(-)$ vs. $\operatorname{COV}(+)$ groups; bolded $\mathrm{P}$ values indicate statistically significant difference. ${ }^{2}$, It indicates the rate of patients who underwent simultaneously SAVR and CABG. SAVR, surgical aortic valve replacement; COV, patients treated before (-) or during (+) coronavirus pandemic; CABG, coronary artery bypass grafting; ACBG, aorto-coronary bypass grafts; CPB, cardio-pulmonary bypass; ACC, aortic-cross clamping; SD, standard deviation. 
Table 4 Serious adverse events in the early postoperative period

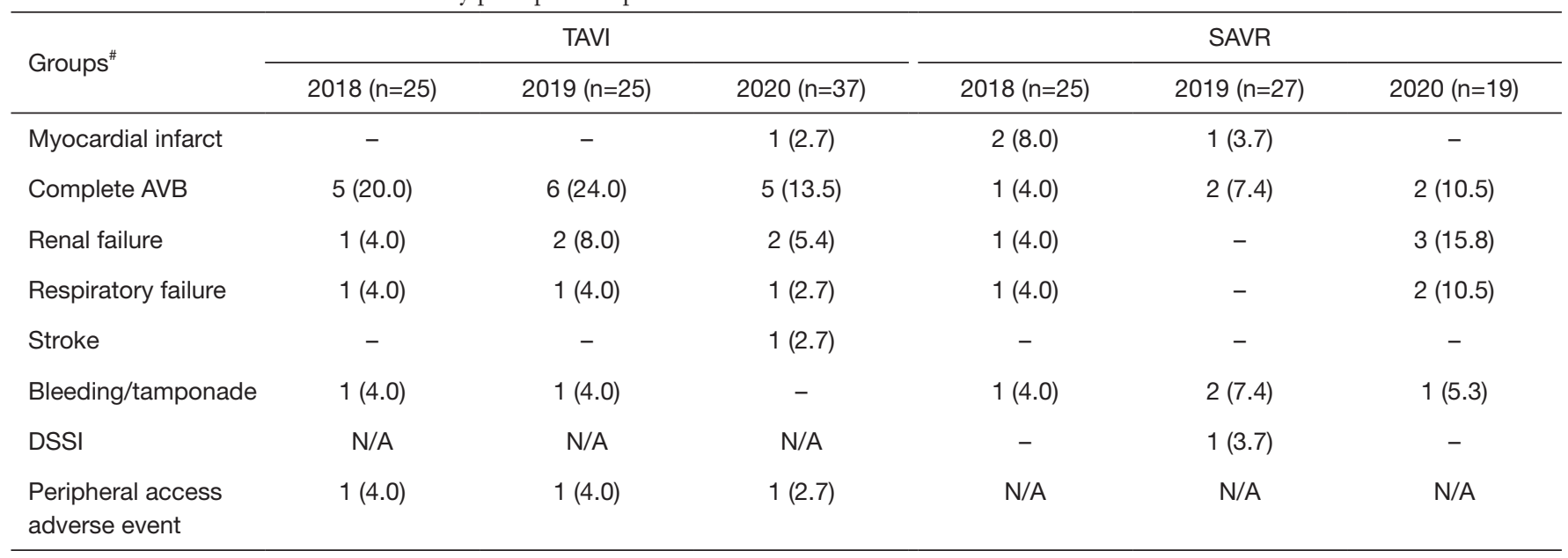

\#, All data are presented as the numbers (n) with percentage (\%), definition of all serious adverse events are listed in the text ('method'). TAVI, transcatheter aortic valve implantation; SAVR, surgical aortic valve replacement; AVB, atrioventricular block; DSSI, deep surgical site infection; N/A, non-applicable.

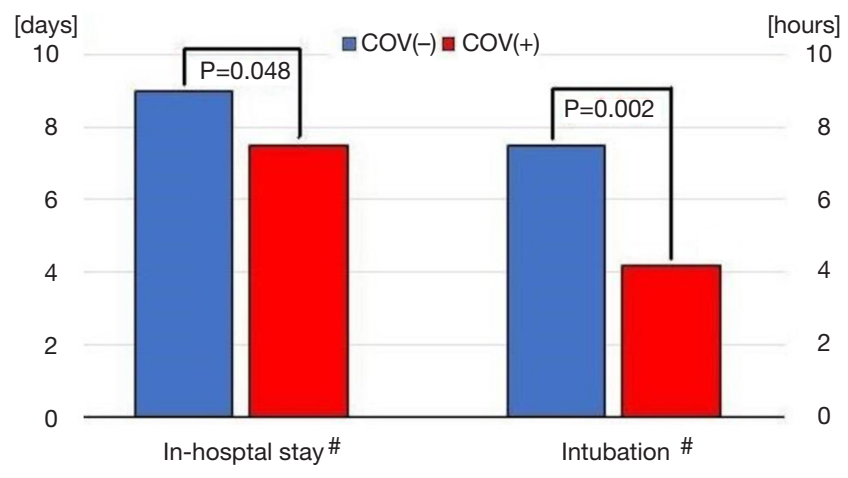

Figure 2 Comparison of intubation time and in-hospital stay length. ", Both continuous variables are expressed as the medians since they have not fulfilled criteria of normal distribution; ${ }^{*}$, it refers to all cases, including those who were not intubated during procedures. $\mathrm{COV}(+) /(-)$, patients who were operated on during $(+)$ or before (-) SARS-CoV-2 pandemic outbreak.

individuals (i.e., TAVI $2018<$ SAVR 2018, TAVI $2019<$ SAVR 2019, TAVI $2020<$ SAVR 2020) (see Table 5).

\section{Discussion}

The main finding in our analysis was a decreased rate of AS patients treated classically in CBP by cardiac surgeons accompanied by an increase in the numbers of TAVI procedures whereas the total number of patients remained unchanged. It is likely that some patients who had TAVI procedures in 2020 would have undergone SAVR in the pre-coronavirus era. However, the tendency to perform more percutaneous cardiac interventions on the aortic valves, at least in the richest countries, is starting to be seen regardless of the COVID-19 pandemic. It seems that in our center, the pandemic had more significant impact of a choice of therapeutic option than a global tendency of TAVI promotion. Herein, the only difference regarding therapeutic method rate was found between 2019 and 2020 but not earlier. In the recent years, more scientific evidence has been accumulated in the clinical trials that TAVI is a safe and efficacious procedure with low complication rates, shorter length of hospital stay, reduced mortality and minimal strokes rate at 30 days as well as years later $(14,15)$. Consequently, according to the current ESC/EACTS guidelines, TAVI can be recommended not only in high but also moderate risk patients (16). Moreover, the recent trials that involved low risk AS subjects, such as PARTNER 3 or EVOLUT, showed better outcomes, including both mortality and morbidity, following TAVI than after SAVR $(17,18)$. The significance of these findings is of crucial importance as most patients (even up to $80 \%$ ) with severe AS are usually at low surgical risk (19). We must be aware that they referred not to all AS subjects but only to those who were appropriate candidates for implantation of biological prostheses. Due to a lack of long-term data on the rate of bioprosthetic structural valve deterioration in younger individuals, low risk but middleaged patients should still undergo SAVR $(16,20)$.

In our opinion, there are a few possible explanations for 
Table 5 Intubation time and in-hospital length in the consecutive years

\begin{tabular}{lccc}
\hline & 2018 & 2019 & 2020 \\
\hline Intubation time (hours) & & & \\
SAVR & $11.5(8.5-13.7)$ & $12.3(10.0-18.6)$ & $12.4(9.1-23.8)$ \\
TAVI & $7.7(3.0-11.9) ; 0(0-5.3)$ & $7.0(4.0-10.0) ; 0(0-4.7)$ & $5.5(2.5-6.8) ; 0(0-3.4)$ \\
P value & 0.001 & $<0.001$ & $<0.001$ \\
In-hospital stay (days) & $13.0(10.5-16.0)$ & $13.0(10.5-14.5)$ & $15.0(11.0-17.0)$ \\
SAVR & $6.0(6.0-8.5)$ & $7.0(5.0-8.0)$ & $7.0(4.0-8.0)$ \\
TAVI & $<0.001$ & $<0.001$ & $<0.001$ \\
P value & & \\
\hline
\end{tabular}

\#, the continuous variables are expressed as the medians with interquartile ranges $\left(25^{\text {th }}-75^{\text {th }}\right.$ percentiles $)$ since they have not satisfied criteria of normal distribution. ${ }^{\&}$, the upper raw refers to TAVI patients who had to be intubated whereas lower one to all TAVI patients. *, It refers to the comparisons of SAVR vs. TAVI [for all patients (lower raw of TAVI subgroup)]. Bolded P values indicate statistically significant difference. SAVR, surgical aortic valve replacement; TAVI, transcatheter aortic valve implantation.

why TAVI procedures were more common in the coronavirus era. Percutaneous techniques are minimally invasive enabling faster recovery and many TAVI cases after careful post-procedure monitoring in the recovery room, may be transferred safely to the cardiology ward (21). As during the pandemic, it is absolutely vital to conserve as many ICU beds or even increase ICU capacity for coronavirus induced hypoxemic respiratory failure patients (22). In addition, innovations in transcatheter heart valve technology, gained experience, smaller sheaths, common application of steadily improving vascular closing devices have resulted in much lower rates of vascular and cardiac complications, reducing a need for ICU admissions (23). Between TAVI and SAVR, the former has a substantially shorter recovery time. Thanks to the experience that we have gained in percutaneous techniques in the last few years, we are able to handle extremely demanding cases (such as after mechanical valve implantations in the mitral position, with a short distance between aortic annulus and coronary ostia, bicuspid aortic valves, moderate dilation of ascending aorta root) as well as shift away from general anesthesia to local with conscious sedation. In the last 3 years, we have had only $20 \%$ of our patients intubated due to either hemodynamic instability or the vascular/cardiac complications of the procedure, comparable with recent reports (24). In our center, conscious sedation is currently the approach of choice as it seems safer and is particularly useful in the coronavirus pandemic. Perioperative viral transmission to the anesthetic team was shown to be reduced if applying just sedation instead of endotracheal intubation and general anesthesia by minimizing the aerosol-generating procedures and reducing the exposure to patient respiratory secretions (25). In many published papers, it can be seen that in experienced centers, in-hospital stay was significantly shorter after TAVI when compared to SAVR (26-30). In addition, SAVR required the application of CPB which is associated with an acute and pronounced inflammatory response consequently activating the complement system and the coagulation pathways. The levels of many pro-inflammatory cytokines such as tumor necrosis factor $\alpha$ as well as interleukin- 6 and -8 (IL-6, IL-8) are high which are proved to be the causative factors of perioperative myocardial and lung injury (31). These increased levels of inflammatory cytokines, particularly IL-6, were also found be correlated with disease severity and mortality in coronavirus induced adult respiratory distress syndrome (ARDS) (32). Therefore, connection of CPB should be avoided in highrisk patients for COVID-19 infection. Of note, only TAVI enables deployment of bioprosthesis on the beating human in CPB. Elderly patients, with many concomitant disorders are considered high risk and the subjects of choice for TAVI.

We also found that patients of higher risk stratified in EuroSCORE II calculator underwent SAVR in the COVID-19 pandemic. It must be stressed that many factors can imply on the final score of perioperative risk (33). Differences in age, NYHA classification, LV performance, and priority of surgery between $\mathrm{COV}(-)$ and $\mathrm{COV}(+)$ SAVR subjects were noted. Although the mean age of subjects in both subgroups were similar, in the $\mathrm{COV}(+)$ subset only one patient was older than 75 years. In 2020, more than one fourth were operated urgently due to severe clinical 
symptoms while in the earlier years only approximately $10 \%$. In the coronavirus era, more symptomatic patients in NYHA III and IV classes with markedly impaired LV systolic performance were operated on. This increase in urgent cases most likely resulted from a fact their clinical status did not allow them to postpone surgery. The vast majority of asymptomatic cases (probably with wellpreserved LVEF) could stay at home and wait for surgery. According to some studies even up to $50 \%$ of all elective cases were cancelled or delayed (34). While beyond the scope of this study, it can be presumed that the operations of many elective patients in good clinical shape and with well-preserved LV systolic function were postponed. It is possible that some of the patients on the waiting list with borderline (e.g., moderate or even low risk, with not so many concomitant diseases) indications for TAVI, eventually underwent percutaneous procedures due to aforementioned limitations regarding access to ICU facilities. On the other hand, it must be consistently kept in mind that severe AS even with trivial symptoms but with hypertrophic myocardium still poses a risk of sudden cardiac death (35). Notable, it was shown previously that nearly $70 \%$ of sudden death episodes that had been reported were not preceded by the classical AS symptoms (36).

Herein, we also observed the changed management with AS patients in the pandemic era did not negatively impact the early clinical outcomes expressed as in-hospital mortality and prevalence of serious adverse events. In our group, overall mortality rate was comparable or slightly higher than in the previously published studies $(37,38)$. Contrary to them, in our group of SAVR cases, even onethirds had to undergo simultaneous CABG $(17,18)$. The latter one was proved to have unfavorable influence on both early and late outcomes, particularly in women (39-41). Moreover, CAD accompanying hypertrophic LV myocardium induced by severe AS poses a high risk of intraoperative myocardial ischemia. Of note, in both patients who died soon after surgery, preoperative coronary angiography revealed significant lesions that had to be addressed during surgery.

Very important issue during the SARS-CoV-2 pandemic is a limited number of beds with monitoring of vital signs. Therefore, currently all actions resulting in the shortening of in-hospital stay are welcome. We did observe the shortest hospitalization time in 2020, mainly due to an increased rate of TAVI procedures. The latter ones, especially these performed on moderate risk patients and with uneventful postprocedural course, were found to be associated with earlier discharge than after SAVR. Our findings regarding this issue supported the previous reports $(17,18,42,43)$. Some of them stressed economic aspects of AS treatment (44). It was showed that more common application of TAVI procedures, in spite of high price of implanted bioprostheses, had important implications in the era of constrained resources with a growing emphasis on reducing health care costs (44).

Furthermore, we are aware of the limitations of this study. First, the number of patients who underwent invasive treatment for severe AS was relatively low and this fact could have impacted the results of the statistical analysis. However, even in this group significant changes were noted. One can not exclude that involving more patients might have been disclosed additional differences between examined periods, for example early mortality. Of note, this is simply a study of a single cardiac surgical center that has had extensive experience in TAVI and most likely not a good representative of the average cardiology ward $(45,46)$. In addition, it must be kept in mind that owing to our experience, some borderline patients were selected for TAVI. Moreover, our hospital is in an example of excellent cooperation between interventional cardiologists and cardiac surgeons that unfortunately is not the rule everywhere. Consequently, a final decision regarding optimal management of AS patients is always the common consensus. As our department was not in the epicenter of the COVID-19 pandemic, a relatively large number of elective patients could be treated. The availability of aortic valve procedures in cardiac centers of hospitals that have been dedicated to the treatment of COVID-19-related severe organ complications is severely reduced.

\section{Conclusions}

The Coronavirus pandemic has changed substantially the management of severe AS. More percutaneous interventions had been performed whereas the open surgical approach was chosen predominantly for urgent patients requiring more complex procedures. This shift into less invasive method of treatment of AS patients resulted in shortening of in-hospital stay without compromise of short-term outcomes. This strategy fulfilled expectations of health care system during SARS-CoV-2 pandemic.

\section{Acknowledgments}

We would like express our thanks to Mr. Konrad Stelmark for editing language of our manuscript. 
Funding: None.

\section{Footnote}

Reporting Checklist: The authors have completed the STROBE reporting checklist. Available at http://dx.doi. org/10.21037/jtd-20-3025

Data Sharing Statement: Available at http://dx.doi. org/10.21037/jtd-20-3025

Peer Review File: Available at http://dx.doi.org/10.21037/jtd20-3025

Conflicts of Interest: All authors have completed the ICMJE uniform disclosure form (available at http://dx.doi. org/10.21037/jtd-20-3025). KR serves as an unpaid editorial board member of Fournal of Thoracic Disease from Aug 2019 to Jul 2021. The other authors have no conflicts of interest to declare.

Ethical Statement: The authors are accountable for all aspects of the work in ensuring that questions related to the accuracy or integrity of any part of the work are appropriately investigated and resolved. The study was conducted in accordance with the Declaration of Helsinki (as revised in 2013). As this study is a retrospective analysis of routine patients treated in the Department of Cardiac Surgery and Transplantology, approval of the Bioethical committee was not necessary and the Institutional Review Board waived the requirement of individual patient consent.

Open Access Statement: This is an Open Access article distributed in accordance with the Creative Commons Attribution-NonCommercial-NoDerivs 4.0 International License (CC BY-NC-ND 4.0), which permits the noncommercial replication and distribution of the article with the strict proviso that no changes or edits are made and the original work is properly cited (including links to both the formal publication through the relevant DOI and the license). See: https://creativecommons.org/licenses/by-nc-nd/4.0/.

\section{References}

1. Carabello BA, Paulus WJ. Aortic stenosis. Lancet 2009;373:956-66.

2. Thaden JJ, Nkomo VT, Enriquez-Sarano M. The global burden of aortic stenosis. Prog Cardiovasc Dis
2014;56:565-71.

3. Zakkar M, Bryan AJ, Angelini AG. Aortic stenosis diagnosis and management. BMJ 2016;355:15425.

4. Jahangiri M, Hussain A, Akowuah E. Minimally invasive surgical aortic valve replacement. Heart 2019;105:s10-5.

5. Ussia GP, Scarabelli $M$, Mulè $M$, et al. Postprocedural management of patients after transcatheter aortic valve implantation procedure with self-expanding bioprosthesis. Catheter Cardiovasc Interv 2010;76:757-66.

6. Qiu H, Tong Z, Ma P, et al. Intensive care during the coronavirus pandemic. Intensive Care Med 2020;46:576-8.

7. Arabi YM, Murthy S, Webb S. COVID-19: a novel coronavirus and a novel challenge for critical care. Intensive Care Med 2020;46:833-6.

8. Li XZ, Jin F, Zhang JG. Treatment of coronavirus disease 2019 in Shandong, China: a cost and affordability analysis. Infect Dis Poverty 2020;9:78.

9. Açikgöz Ö, Günay A. The early impact of the Covid-19 pandemic on the global and Turkish economy. Turk J Med Sci 2020;50:520-6.

10. Giordano A, Biondi-Zoccai A, Frati G, et al. Management of structural heart disease and acute coronary syndrome in the COVID-19 pandemic. Curr Atheroscler Rep 2020;22:29.

11. Płońska-Gościniak E, Suwalski $\mathrm{P}$, Bartuś $\mathrm{S}$, et al. Management of valvular and structural heart diseases during the coronavirus disease 2019 pandemic: an expert opinion of the Working Group on Valvular Heart Diseases, the Working Group on Cardiac Surgery, and the Association of Cardiovascular Interventions of the Polish Cardiac Society. Kardiol Pol 2020;78:498-507.

12. Fudulu DP, Angelini GD. Cardiac Surgery in the time of the coronavirus. J Card Surg 2020;35:1177-9.

13. Komosa A, Perek B, Rzymski P, et al. Transcatheter aortic valve replacement is associated with less oxidative stress and faster recovery of antioxidant capacity than surgical aortic valve replacement. J Clin Med 2019;8:1364.

14. Waksman R, Corso PJ, Torguson R, et al. TAVR in lowrisk patients: 1-year results from the LRT Trial. JACC Cardiovasc Interv 2019;12:901-7.

15. Barbanti M, van Mourik MS, Spence MS, et al. Optimising patient discharge management after transfemoral transcatheter aortic valve implantation: the multicentre European FAST-TAVI trial. EuroIntervention 2019;15:147-54.

16. Baumgartner H, Falk V, Bax JJ, et al. 2017 ESC/EACTS Guidelines for the management of valvular heart disease. Eur Heart J 2017;38:2739-91. 
17. Mack MJ, Leon MB, Thourani VH, et al., on behalf of the PARTNER 3 Investigators. Transcatheter aortic-valve replacement with a balloon-expandable valve in low-risk patients. N Engl J Med 2019;380:1695-705.

18. Popma JJ, Deeb GM, Yakubov SJ, et al. Transcatheter aortic-valve replacement with a self-expanding valve in low-risk patients. N Engl J Med 2019;380:1706-15.

19. Thourani VH, Suri MR, Gunter RL, et al. Contemporary real-world outcomes of surgical aortic valve replacement in 141,905 low-risk, intermediate-risk, and high-risk patients. Ann Thorac Surg 2015;99:55-61.

20. Siddique S, Gada H, Mumtaz MA, et al. Should all lowrisk patients now be considered for TAVR? Operative risk, clinical, and anatomic considerations. Curr Cardiol Rep 2019;21:161.

21. Leclercq F, Iemmi A, Lattuca B, et al. Feasibility and safety of transcatheter aortic valve implantation performed without intensive care unit admission. Am J Cardiol 2016;118:99-106.

22. Phua J, Weng L, Long L, et al. Intensive care management of coronavirus disease 2019 (COVID-19): challenges and recommendations. Lancet Respir Med 2020;8:506-17.

23. Akodad M, Lefèvre T. TAVI: Simplification is the ultimate sophistication. Front Cardiovasc Med 2018;5:96.

24. Mayr NP, Michel J, Bleiziffer S, et al. Sedation or general anesthesia for transcatheter aortic valve implantation (TAVI). J Thorac Dis 2015;7:1518-26.

25. Lie SA, Wong SW, Wong LT, et al. Practical considerations for performing regional anesthesia: lessons learned from the COVID-19 pandemic. Can J Anaesth 2020;67:885-92.

26. Akintoye E, Ando T, Sandio A, et al. Aortic valve replacement for severe aortic stenosis before and during the era of transcatheter aortic valve implantation. Am J Cardiol 2020;126:73-81.

27. Mäkikallio T, Jalava MP, Husso A, et al. Ten-year experience with transcatheter and surgical aortic valve replacement in Finland. Ann Med 2019;51:270-9.

28. Calle-Valda CM, Aguilar R, Benedicto A, et al. Outcomes of aortic valve replacement according to surgical approach in intermediate and low risk patients: a propensity score analysis. Heart Lung Circ 2018;27:885-92.

29. Burrage $M$, Moore $P$, Cole C, et al. Transcatheter aortic valve replacement is associated with comparable clinical outcomes to open aortic valve surgery but with a reduced length of in patient hospital stay: a systematic review and meta-analysis of randomised trials. Heart Lung Circ 2017;26:285-95.
30. Gaudriot B, Uhel F, Gregoire M, et al. Immune dysfunction after cardiac surgery with cardiopulmonary bypass: beneficial effects of maintaining mechanical ventilation. Shock 2015;44:228-33.

31. Giomarelli P, Scolletta S, Borrelli E, et al. Myocardial and lung injury after cardiopulmonary bypass: role of interleukin (IL)-10. Ann Thorac Surg 2003;76:117-23.

32. McGonagle D, Sharif K, O'Regan A, et al. The role of cytokines including interleukin-6 in COVID-19 induced pneumonia and macrophage activation syndrome-like disease. Autoimmun Rev 2020;19:102537.

33. Ad N, Holmes SD, Patel J, et al. Comparison of EuroSCORE II, original EuroSCORE, and The Society of Thoracic Surgeons Risk Score in cardiac surgery patients. Ann Thorac Surg 2016;102:573-9.

34. Mehta JJ, Patel J, Ayoub B, et al. Caution regarding potential changes in AVR practices during the COVID-19 pandemic. J Card Surg 2020;35:1168-9.

35. Taniguchi T, Morimoto T, Shiomi H, et al. Sudden death in patients with severe aortic stenosis: observations from the CURRENT AS Registry. J Am Heart Assoc 2018;7:e008397.

36. Généreux P, Stone GW, O'Gara PT, et al. Natural history, diagnostic approaches, and therapeutic strategies for patients with asymptomatic severe aortic stenosis. J Am Coll Cardiol 2016;67:2263-88.

37. Eggebrecht H, Bestehorn K, Rassaf T, et al. In-hospital outcomes after transcatheter or surgical aortic valve replacement in younger patients less than 75 years old: a propensity-matched comparison, EuroIntervention 2018;14:50-7.

38. Kolte D, Vlahakes GJ, Palacios IF, et al. Transcatheter versus surgical aortic valve replacement in low-risk patients. J Am Coll Cardiol 2019;74:1532-40.

39. Sasaki Y, Hirai H, Hosono M, et al. Adding coronary artery bypass grafting to aortic valve replacement increases operative mortality for elderly (70 years and older) patients with aortic stenosis. Gen Thorac Cardiovasc Surg 2013;61:626-31.

40. Perek B, Misterski M, Stachowiak W, et al. The impact of coronary artery disease severity on late survival after combined aortic valve replacement and coronary artery bypass grafting - experience of a single cardiac surgery center. Kardiochir Torakochirurgia. Pol 2014;11:361-6.

41. Guedeney P, Tchétché D, Petronio AS, et al. Impact of coronary artery disease and percutaneous coronary intervention in women undergoing transcatheter aortic valve replacement: From the WIN-TAVI registry. 
Catheter Cardiovasc Interv 2019;93:1124-31.

42. Serletis-Bizios A, Durand E, Cellier G, et al. A prospective analysis of early discharge after transfemoral transcatheter aortic valve implantation. Am J Cardiol 2016;118:866-72.

43. Elbadawi A, Saad M, Elgendy IY, et al. Temporal trends and outcomes of transcatheter versus surgical aortic valve replacement for bicuspid aortic valve stenosis. JACC Cardiovasc Interv 2019;12:1811-22.

44. Arora S, Strassle PD, Kolte D, et al. Length of stay and discharge disposition after transcatheter versus surgical

Cite this article as: Perek B, Olasinska-Wisniewska A, Misterski M, Puslecki M, Grygier M, Buczkowski P, Lesiak M, Stankowski T, Szarpak L, Ruetzler K, Turan O, Jemielity M. How the COVID-19 pandemic changed treatment of severe aortic stenosis: a single cardiac center experience. J Thorac Dis 2021;13(2):906-917. doi: 10.21037/jtd-20-3025 aortic valve replacement in the United States. Circ Cardiovasc Interv 2018;11:e006929.

45. Grygier M, Misterski M, Araszkiewicz A, et al. First implantation of the new Lotus Edge transcatheter aortic valve in Poland. Kardiol Pol 2019;77:1084-6.

46. Olasinska-Wisniewska A, Grygier M, Lesiak M, et al. Short- and mid-term outcome of transcatheter aortic valve implantation in patients with advanced age. Cardiol J 2017;24:358-63. 